\title{
Mapping Rumen Microbial Complexity through '-Omics'
}

\author{
Durgadevi Aphale ${ }^{1,2}$, Sharad Laldas ${ }^{1}$ and Aarohi Kulkarni ${ }^{1 *}$ \\ ${ }^{1}$ Praj Matrix, R\&D Center, Division of Praj Industries Ltd, India \\ ${ }^{2}$ Department of Health and Biological Sciences, Symbiosis International Deemed University, India
}

Submission: May 16, 2019; Published: June 14, 2019

"Corresponding author: Aarohi Kulkarni, Praj Matrix, R\&D Center, Division of Praj Industries Ltd, 402/403/1098, Urawade, At: Pirangut; Mulshi, Pune, Maharashtra, India

\begin{abstract}
The rumen is a highly complex and dynamic ecosystem where, the microbiome displays symbiotic interactions and provides energy to ruminants through breakdown of plant fibers. The current study hypothesized that; microbiome deciphers niche specialization for nutrient utilization and engineers the rumen ecosystem comprehensively. It has raised the interest in fundamental understanding of the rumen microbiome to develop novel approaches for improving the livestock production. Present mini-review with current literature reveals upcoming 'omics' based approaches in order to ascertain the said purpose and would accomplish the major progress in animal phenotype improvement through Rumen microbiome understanding and their manipulation.
\end{abstract}

Keywords: Omics; Rumen; Live-stock health; Rumen microbiome; Biomarkers; Proteins; Lipids

Abbreviations: ARDRA: Amplified Ribosomal DNA Restriction Analysis; FISH: Fluorescence In Situ Hybridization; DGGE: Denaturing Gradient Gel Electrophoresis; SSCP: Single-Strand Conformation polymorphism; RAPD: Random Amplified Polymorphic DNA; DAF: DNA Amplification Fingerprinting; ARISA: Automated Ribosomal Intergenic Spacer Analysis; T-RFLP:Terminal Restriction Fragment Length Polymorphism; qPCR: quantitative PCR

\section{Introduction}

The exploration of relationship between the rumen fluid, bacteria and fiber digestion by [1-3] could isolate 200 microbial species by cultivation-based approaches, majorly using roll tube technique. It represented only about $10-20 \%$ of the total rumen microbiome thus, underestimating the true microbial diversity. A research by [4] states about prime functionalities of cultivable and uncultivable rumen microbiome in providing metabolic energy to the host animal where 1000 times more genes of rumen microbiome results into hydrolysis and fermentation of inaccessible nutrients provided to the ruminant. Therefore, it is critical to understand the complex interplay of the rumen microbiome, the host animal and the external influencing environment in order to escalate wide opportunities for improving live-stock health and productivity. Development of unique biomarkers, proteins, lipids, DNA and RNA as different units of the study are necessary for the same [5].

It further resulted into emergence of small subunit ribosomal RNA (rRNA) as the most significant tool to understand the rumen ecology. In this conjunction, 16S rDNA gene cloning approach contributed to exploration of rumen microbial diversity [6], though it couldn't explore the microbial dynamics including the effects of environmental perturbations on microbial ecosystem. Then after, techniques including Amplified Ribosomal DNA Restriction Analysis (ARDRA) [7], blotting, Fluorescence In Situ Hybridization (FISH), Denaturing Gradient Gel Electrophoresis (DGGE),single-strand conformation polymorphism (SSCP) [8], Random Amplified Polymorphic DNA (RAPD), DNA amplification fingerprinting (DAF) [9], Automated Ribosomal Intergenic Spacer Analysis (ARISA) [10], DNA microarrays [11], terminal restriction fragment length polymorphism (T-RFLP) [12], length heterogeneity PCR [13] and quantitative PCR (qPCR) [14] changed the rumen microbiology era that greatly explored the rumen microbial community's abundance, expressions and diversity in an absolute way. However, the techniques represented pitfall in exploration of total microbiota from a particular niche at a greater depth, effect of environmental factors on microbiota and their interaction with others.

It led to a commencement of metagenomics, metatranscriptomics, proteomics and large-scale metabolomics studies [15-18] which are being endeavored in the cattle rumen recently [16]. The study inferred that; low abundant taxa may have significant role in the host physiology nullifying the concept 
of abundance based functional significance [19]. In addition, focus of rumen microbiome contribution to agricultural greenhouse gas emission is shifted towards defining the rumen microbiota of efficient ruminants especially for meat and dairy sectors and studying an influence of the host genetics on molding the rumen microbiome [20]. This mini-review summarizes the current omics based approaches to delve into the rumen microbial community and thereby to predict various functional levels; including gene abundance and expression, protein expression, metabolite profile and the level or intensity of metabolites that would build a tangible platform for remarkable improvement in live-stock health and production sector in coming future.

\section{Brief description of the current omics approaches for decoding the rumen microbiome}

Limitations of traditional rumen cultivation approaches have given new way for omics; non-cultivation-based methodologies, though its use in livestock is at preliminary level, especially for ruminants [21]. Studies were conducted towards exploration of taxonomic changes under dietery interventions. A recent global effort revealed core, diet-driven rumen microbiome using more than 700 samples [22]. Therefore, global research predicts that, microbial markers of feed efficiency can be identified through in-depth omics analyses of the rumen microbiome. In specific, metagenomics can aid in driving microbial fermentation according to microbial metabolic capacities thereby utilizing the available feed substrates more effectively [23] towards improved meat and milk production. Further, meta transcriptomic approaches can unfold the expression of fiber-degrading genes and their pattern variations with external interventions. The better understanding of their metabolic capacity can serve as a backbone in designing and optimizing the feed or external supplement (probiotics) ratios, to maximize the rumen fermentation towards high productivity. The protein expression analysis through meta-proteomics revealed over 2,000 proteins associated with rumen microbiome [24]. The development of Meta-proteome Analyzer software can also aid in the analysis and interpretation of meta-protein data [25]. The integration of sequencing and metabolomics can provide a more comprehensive analysis of the rumen system that would contribute in gathering information regarding metabolic expression of the genetic potential of the microbiome [26]. In addition, Culturomics is yet crucial for improving the reference database for ruminomics studies in order to have in vitro and in vivo experimentation in place as per the requirements. The following sections summarize individually the various omics-based approaches involved in the rumen biotechnological studies.

Metagenomics: Metagenomics conquered the necessity of microbial culturing and thus became a critical approach towards exploration of full genomic potential residing inside the rumen microbiome. Xue et al. [27] predicted 27,555 genes and 90 different proteins through metagenome technology; $57 \%$ of which had association with fiber hydrolysis. Metagenomics currently offers the ability of whole and fragmented genomes assemblage, gene prediction, mapping and discovering of enzymes and pathways, quantification of abundance of functional genomic elements across and between samples leading to exemplary insights of rumen metagenome [5]. The advancement of sequencing technology confers greater depth in sequencing, where bioinformatic algorithms are used to generate kmer patterns unique to each microbe that sort the metagenomic fragments [28]. The accuracy of metagenomics is based on improved algorithms and shall have future contribution from Hungate 1000 project.

Met-transcriptomics: An expression-based omics approach; the Meta-transcriptomics involves the profiling of communitywide expressed genes (mRNA), also termed as RNA-seq. The approach permits evaluation of diversity and predicts functional capacity of the microbiome, providing insights into actual microbiome function via gene expression [5]. Furthermore; MetaRibo-Seq a ribosome profiling, tool that is currently at infancy level of meta-transcriptomics = demands validation of rumen microbiome and has emerged due to weak co-relation between mRNA and protein levels during meta-transcriptomics analytics.

Metaproteomics: It is an emerging technology, termed as the entire protein complement of the microbiota in an environment at a given point in time; that stands between the established DNA, RNA sequencing and metabolomics approach in order to characterize the functional activity of the rumen microbiome (29). Due to the tedious processing and challenges, very few reports focused on rumen meta-proteomics. In the current date, development of next generation mass spectrometers and software enabled protein identification en masse using the approach similar to shotgun sequencing. In this context, [24] deciphered over 2000 proteins associated with rumen microbiome. Also, the current approach by Hart [29] states about comparison between meta-proteomic data and metatranscriptomics information, that can decode various proteins associated with rumen functionalities. The prime software Metaproteome Analyzer can aid to infer the meta-protein data [25].

Metabolomics: Metabolomics is defined as the comprehensive analysis of metabolites from microbiome or biological system in qualitative and quantitative ways [30]. There are very few reports available on rumen metabolome research. Metabolomics mainly targets low molecular mass molecules $(<1,000 \mathrm{Da})$, that can be functionally assigned [31]. The inferences of metabolomics are based on the usage of analytical techniques including; LC-MS, GC-MS, NMR etc. The combinatorial studies between sequencing and metabolomics are uncommon and can provide diligent analysis of the rumen system, regarding metabolic expression of the genetic potential [26].

Culturomics: Since the Hungate 1966 project, technology has rapidly advanced, to establish the backbone of Hungate 1000 project [32] led by AgResearch, New Zealand. This is targeted 
towards genome sequencing of 1000 rumen strains, out of which, sequencing of 420 genomes has been concluded, representing only 117 bacterial species and $3.6 \%$ of operational taxonomic units from RDP database [33]. Furthermore; Rumen Microbial Genomics (RGM) network was established in 2011, in order to comprehend the synergistic mechanisms of rumen microbiome and improvement of reference databases to isolate yet uncultured microorganisms. The culturing ability of rumen microbiome has been expanded through research work conducted by [34] and [35]. Indeed, there are many technologies emerging towards cultivation of yet unculturable rumen bacteria.

\section{Conclusion}

The rumen environment contains multitude of molecules of microbial, plant and animal origins. The '-omics' based rumen characterization would comprehend the rumen microbiome and their interactions, functionalities, pathways and proteins associated therein. The focus of exploration of rumen ecological foundation may deliver improved apprehension of rumen, novel molecules and unexplored metabolic pathways that would lead to insights of this vast community. Key focused interventions based on outcomes of omics approaches could lead to fulfillment of worldwide demands of ruminant meat and milk.

\section{References}

1. Smith, PH, Hungate RE (1958) Isolation and Characterization of Methanobacterium ruminantium n. sp. J Bacteriol 75: 713-718.

2. Bladen HA, Bryant MP, Doetsch RN (1961) A Study of Bacterial Species from the Rumen which Produce Ammonia from Protein Hydrolyzate. Appl Microbiol 9: 175-180.

3. Chung KT, Bryant MP (1997) Robert E. Hungate: Pioneer of Anaerobic Microbial Ecology. Anaerobe 3: 213-217.

4. Shabat SK, Sasson G, Doron-Faigenboim A, Durman T, Yaacoby S, et al. (2016) Specific Microbiome-dependent Mechanism underlie the Energy Harvest Efficiency of Ruminants. ISME J 10: 2958-2972.

5. Ward, DM, Weller R, Bateson MM (1990) 16S rRNA Sequences Reveal Numerous Uncultured Microorganisms in a Natural Community. Nature 345(6270): 63-65.

6. Smith E, Leeflang P, Wernars K (1997) Detection of Shifts in Microbial Community Structure and Diversity in Soil caused by Copper Contamination using Amplified Ribosomal DNA Restriction Analysis. FEMS Microbiol Ecol 23: 249-261.

7. Schwieger F, Tebbe CC (1998) A New Approach to utilize PCR SingleStrand Conformation Polymorphism for 16S rRNA Gene-based Microbial Community Analysis. Appl Environ Microbiol 64: 4870-4876.

8. Franklin RB, Taylor DR, Mills AL (1999) Characterization of Microbial Communities using Randomly Amplified Polymorphic DNA (RAPD). J Microbiol Methods 35: 225-235.

9. Fisher MM, Triplett EW (1999) Automated Approach for Ribosomal Intergenic Spacer Analysis of Microbial Diversity and its Application to Freshwater Bacterial Communities. Appl Environ Microbiol 65: 46304636.

10. Gentry T, Wickham G, Schadt C, He Z, Zhou J (2006) Microarray Applications in Microbial Ecology Research. Microbiol Ecol 52: 159175.

11. Thies JE (2007) Soil Microbial Community Analysis using Terminal restriction Length Polymorphisms. Soil Sci Soc Am 71: 579-591.
12. Mills DK, Entry JA, Gillevet PM, Mathee K (2007) Assessing Microbial Community Diversity using Amplicon Length Heterogeneity Polymerase Chain Reaction. Soil Sci Soc Am J 71: 572-578.

13. Smith CJ, Osborn AM (2009) Advantages and Limitations of Quantitative PCR (Q-PCR)-based Approaches in Microbial Ecology. FEMS Microbiol Ecol 67: 6-20.

14. Morgavi DP, Rathahao-Paris E, Popova M, Boccard J, Nielsen KF, et al. (2015) Rumen Microbial Communities Influence Metabolic Phenotypes in Lambs. Front Microbiol 6: 1060.

15. Wallace RJ, Rooke JA, McKain N, Duthie CA, Hyslop JJ, et al. (2015) The Rumen Microbial Metagenome associated with High Methane Production in Cattle. BMC Genomics 16: 839.

16. Weimer PJ, Nerdahl M, Brandl DJ (2015) Production of Medium-Chain Volatile Fatty Acids by Mixed Ruminal Microorganisms is enhanced by Ethanol in Co-culture with Clostridium kluyeri. Bioresour Technol 175: 97-101.

17. Huws SA, Edwards JE, Creevey CJ, Stevens P, Lin W, et al. (2016) Temporal Dynamics of the Metabolically Active Rumen Bacteria Colonizing Fresh Perennial Ryegrass. FEMS Microbiol Ecol 92: 137.

18. Arumugam M, Raes E, Pelletier D, Paslier T, Yamada DR, et al. (2011) Enterotypes of the Human Gut Microbiome. Nature 473(7346): 174180.

19. Jami E, White BA, Mizrahi I (2014) Potential Role of the Bovine Rumen Microbiome in Modulating Milk Composition and Feed Efficiency. Plosone 9: e85423.

20. Snelling TJ, Wallace RJ (2017) The Rumen Microbial Metaproteome as revealed by SDS-PAGE. BMC Microbiol 17(1): 9.

21. Henderson GF, Cox S, Ganesh A, Jonker W, Young Global Rumen Census Collaborators, et al. (2015) Rumen Microbial Community Composition varies with Diet and Host, but a Core Microbiome is found across a Wide Geographical Range. Sci Rep 5: 14567.

22. Eisler MC, Lee JF, Tarlton GB, Martin J, Beddington JA, et al. (2014) Agriculture: Steps to Sustainable Livestock. Nature 507(7490): 32-34.

23. Deusch S, Seifert J (2015) Catching the Tip of the Iceberg-Evaluation of Sample Preparation Protocols for Metaproteomic Studies of the Rumen Microbiota. Proteomics 15: 3590-3595.

24. Muth T, Behne A, Heyer R, Kohrs F, Benndorf D, et al. (2015) The Metaproteomeanalyzer: A Powerful Open-Source Software Suite for Metaproteomics Data Analysis and Interpretation. J Proteome Res 14: 1557-1565.

25. O'callaghan T, Vazquez-Fresno R, Serra-Cayuela A, Dong E, Mandal R, et al. (2018) Pasture Feeding Changes the Bovine Rumen and Milk Metabolome. Metabolites 8: 27.

26. Xue F, Nan X, Pan X, Zheng S, Jiang L, et al. (2017) Application of Multi Omics Technologies in Ruminants Research. J Dairy Vet Sci 1(3). 555563.

27. Huws SA, Creevey CJ, Oyama LB, Mizrahi I, Denman SE, et al. (2018) Addressing Global Ruminant Agricultural Challenges Through Understanding the Rumen Microbiome: Past, Present, and Future. Front Microbiol 9: 2161.

28. Droge J, McHardy AC (2012) Taxonomic Binnig of Metagenomic Samples generated by Next-Generation Sequencing Technologies. Briefings in Bioinformatic 13(6): 646-655.

29. Hart EH, Creevey CJ, Hitch T, Kingston-Smith AH (2018) Metaproteomics of Rumen Microbiota Indicates Niche Compartmentalisation and Functional Dominance in a Limited Number of Metabolic Pathways between Abundant Bacteria. Sci Rep 8: 10504.

30. Yi L, Shi S, Wang Y, Huang W, Xia ZA, et al. (2016) Serum Metabolic Profiling reveals Altered Metabolic Pathways in Patients with Posttraumatic Cognitive Impairments. Sci Rep 6: 21320. 
31. Bundy JG, Davey MP, Viant MR (2009) Environmental Metabolomics: A Critical Review and Future Perspectives. Metabolism 5:3

32. Seshadri R, Leahy SC, Attwood GT, Teh KH, Lambie SC, et al. (2018) Cultivation and Sequencing of Rumen Microbiome Members from the Hungate1000 Collection. Nat Biotech 36: 359.

33. Zehavi T, Probst M, Mizrahi I (2018) Insights into the Culturomics of the Rumen Microbiome. Front Microbiol 9: 1999.
34. Kenters N, Henderson G, Jeyanathan J, Kittelmann S, Janssen PH (2011) Isolation of Previously Uncultured Rumen Bacteria by Dilution to Extinction using a New Liquid Culture Medium. J Microbiol Meth 84: $52-60$

35. Poelaert C, Nollevaux G, Boudry C, Taminiau B, Nezer C, et al. (2018) Reducing Agent can be omitted in the Incubation Medium of the Batch in vitro Fermentation Model of the Pig Intestines. Animal 12: 11541164

\section{Your next submission with Juniper Publishers will reach you the below assets}

- Quality Editorial service

- Swift Peer Review

- Reprints availability

- E-prints Service

- Manuscript Podcast for convenient understanding

- Global attainment for your research

- Manuscript accessibility in different formats ( Pdf, E-pub, Full Text, Audio)

- Unceasing customer service

Track the below URL for one-step submission https://juniperpublishers.com/online-submission.php 